\title{
Circulations, Fuzzy Relations and Semirings
}

\author{
Roland Glück and Bernhard Möller \\ Institut für Informatik, Universität Augsburg, \\ Universitätsstr. 14, D-86135 Augsburg, Germany \\ \{glueck, moeller\}@informatik. uni-augsburg.de \\ http://www.informatik. uni-augsburg.de/en/chairs/dbis/ \\ $\mathrm{pmi} / \mathrm{staff} /\{$ glueck, moeller $\}$
}

\begin{abstract}
Circulations are similar to flows in capacity-constrained networks, with the difference that they also observe lower bounds and, unlike flows, are not directed from a source to a sink. We give a new description of circulations in networks using a technique introduced by Kawahara; he applied the same methods to network flows. We show the power and flexibility of his approach in a new application, refining it at the same time by introducing the concept of test relations. Furthermore we will give algebraic formulations of a generic algorithm for computing a flow in a network with lower bounds and a sufficient and necessary criterion for the existence of a circulation.
\end{abstract}

\section{Introduction}

Networks with and without lower bounds as well as flows and circulations in them have a wide range of applications. They are used for transport problems, for modelling financial and economic situations and are also used in graph theory. Common proofs of Hall's theorem and of the Egervary-König-Theorem ([Jun], Chapter 7) use networks, too. Also problems concerning matchings in bipartite graphs and disjoint path problems can often be solved using networks. So there are a lot of algorithms for and theorems about them.

Usually networks are described as graphs with weighted edges. In the approach we are using they are modelled as so-called fuzzy relations, a natural generalisation of traditional relations. This idea was introduced in [Kaw]. The advantage of this approach is that a lot of proofs can be done in an algebraic manner by simple calculation. This opens the door for automated reasoning about networks and related topics in graph theory.

We take up Kawahara's approach in a new application. At the same time we refine it by introducing the concept of fuzzy test relations, inspired by the theory of tests in semirings [KozT,MB], which leads to a substantial notational and conceptual simplification. This will be beneficial for automated proofs in this problem domain.

In Section two we present the basics of fuzzy relations and other tools we will use. Section three gives an overview over recent algebraic work concerning flows in networks. Finally, from Section four on we present our new ideas about circulations in networks with lower bounds. 


\section{Fuzzy Relations}

\subsection{Definition and Basic Operations}

Definition 2.1 (Fuzzy Relation). A fuzzy relation $\alpha$ between sets $X$ and $Y$, written $\alpha: X \leftrightarrow Y$, is a mapping from $X \times Y$ into the interval [0,1]. The set of all fuzzy relations between $X$ and $Y$ is denoted by $\operatorname{Rel}(X, Y)$. A fuzzy relation between a set $X$ and itself is called a fuzzy endorelation on $X$.

A fuzzy endorelation $\alpha$ on a set $X$ can be viewed as representing a weighted directed graph with node set $X$, where for $x, y \in X$ the value $\alpha(x, y)$ is the weight of edge $(x, y)$. An edge weight $\alpha(x, y)=0$ means that $x$ and $y$ are considered not to be connected by a direct edge. The notion of a fuzzy relation can be generalised to allow elements of an arbitrary lattice as edge weights. However, the above special case has a number of advantages in our setting; they will be discussed as we go along.

Definition 2.2 (Special Relations). Given sets $X$ and $Y$, there are three particular fuzzy relations with

$$
\begin{array}{ll}
\mathbf{0}_{X Y}: X \leftrightarrow Y, & \mathbf{0}(x, y)=0, \\
\nabla_{X Y}: X \leftrightarrow Y, & \nabla(x, y)=1 \\
i d_{X}: X \leftrightarrow X, & i d_{X}(x, y)=\left\{\begin{array}{l}
1 \text { if } x=y \\
0 \text { otherwise }
\end{array}\right.
\end{array}
$$

for all $x, y$, called the empty, universal and identity fuzzy relation, resp. When $X$ and $Y$ are clear from the context the indices will be omitted.

In the graph view, $\mathbf{0}$ represents the totally disconnected graph while $\nabla$ is the fully connected graph in which all edges have maximal weight. Finally, $i d$ is a graph in which every node carries a loop of maximal weight and there are no other edges.

Definition 2.3 (Boolean Fuzzy Relation). A fuzzy relation is called Boolean if its range is contained in the set $\{0,1\}$.

A Boolean fuzzy relation $\alpha: X \leftrightarrow Y$ corresponds in a natural way to a standard relation $\widetilde{\alpha} \subseteq X \times Y$ given by

$$
x \widetilde{\alpha} y \Leftrightarrow \alpha(x, y)=1 .
$$

To motivate the following definitions we will now explain how fuzzy relations can be used to model flows and circulations.

A network is an edge-labeled directed graph with at most one edge between any two nodes, in which the edge labels are non-negative real numbers modelling transport capacities between the nodes. If there is an upper bound $c \neq 0$ for the capacities (which holds, in particular, for finite networks) we can normalise them to the interval $[0,1]$ by dividing them by $c$. Now the network can immediately 
be represented by a fuzzy relation $\alpha$, which takes value $\frac{d}{c}$ if there is an edge with weight $d$ from $x$ to $y$ and zero otherwise.

It is well known how to use standard relations for describing certain aspects of graphs. We want to extend these techniques to networks and their describing fuzzy relations.

To this end we first introduce some operations on the interval $[0,1]$. For $a, b \in[0,1]$ we define

- $a \vee b=\max \{a, b\}$

- $a \wedge b=\min \{a, b\}$

- $a \ominus b=\max \{0, a-b\}$

- $a \oplus b=\min \{1, a+b\}$

Consistent with these definitions we define the operators $\bigvee_{x \in X} x$ and $\bigwedge_{x \in X} x$ for arbitrary subsets $X \subseteq[0,1]$ by $\bigvee_{x \in X} x=\sup X$ and $\bigwedge_{x \in X} x=\inf X$.

Definition 2.4 (Basic Operations). For fuzzy relations $\alpha, \beta: X \leftrightarrow Y$ the join $\alpha \sqcup \beta$, the meet $\alpha \sqcap \beta$, the truncating difference $\alpha \ominus \beta$ and the truncating sum $\alpha \oplus \beta: X \leftrightarrow Y$ are the pointwise extensions of the operators defined above:

- $\forall x \in X \forall y \in Y:(\alpha \sqcup \beta)(x, y)=\alpha(x, y) \vee \beta(x, y)$

- $\forall x \in X \forall y \in Y:(\alpha \sqcap \beta)(x, y)=\alpha(x, y) \wedge \beta(x, y)$

- $\forall x \in X \forall y \in Y:(\alpha \ominus \beta)(x, y)=\alpha(x, y) \ominus \beta(x, y)$

- $\forall x \in X \forall y \in Y:(\alpha \oplus \beta)(x, y)=\alpha(x, y) \oplus \beta(x, y)$

Two fuzzy relations $\alpha, \beta: X \leftrightarrow Y$ are said to be disjoint if $\alpha \sqcap \beta=\mathbf{0}$. Adapting the usual notation $A \dot{\cup} B$ for the union of disjoint sets $A$ and $B$, we abbreviate the union of two disjoint fuzzy relations $\alpha$ and $\beta$ by $\alpha \dot{\sqcup} \beta$. Moreover, we write $\alpha \sqsubseteq \beta$ if $\alpha(x, y) \leq \beta(x, y)$ holds for all $(x, y) \in X \times Y$.

Analogously to above we extend $\sqcup$ to sets of fuzzy relations, writing $\bigsqcup_{i \in I} \alpha_{i}$.

It is easy to see that meet, join and truncating sum are commutative and associative. Moreover, join distributes over meet and vice versa.

The behaviour of the truncating sum is more complex. Due to the truncation the common laws of addition and subtraction are not transferable. With additional assumptions similar rules are valid. For example $\beta \sqsubseteq \alpha$ implies $(\alpha \ominus \beta) \oplus \beta=\alpha$. In Section 2.3 we will state similar properties concerning the connection between the above operations and the cardinality of fuzzy relations.

Definition 2.5 (Scalar Multiplication). For real numbers $k \in[0,1]$ and a fuzzy relation $\alpha: X \leftrightarrow Y$ the scalar multiplication $k \cdot \alpha$, also written $k \alpha$, is defined by $(k \alpha)(x, y)=k \cdot \alpha(x, y)$ for all $(x, y) \in X \times Y$.

Scalar multiplication distributes over $\sqcup, \sqcap, \ominus$ and $\oplus$.

Definition 2.6 (Converse). For a fuzzy relation $\alpha: X \leftrightarrow Y$ the converse $\alpha^{\sharp}: Y \leftrightarrow X$ is defined by

$$
\forall x \in X \forall y \in Y: \alpha^{\sharp}(y, x)=\alpha(x, y) .
$$

Converse commutes with scalar multiplication, i.e., $(k \alpha)^{\sharp}=k\left(\alpha^{\sharp}\right)$, and distributes over $\sqcup, \sqcap, \ominus$ and $\oplus$. In the graph view this operation reverses all edges while preserving their weights. 


\subsection{Composition, Powers and Star}

Definition 2.7 (Composition). We define the composition $\alpha \beta: X \leftrightarrow Z$ of two fuzzy relations $\alpha: X \leftrightarrow Y$ and $\beta: Y \leftrightarrow Z$ by

$$
\alpha \beta(x, z)=\bigvee_{y \in Y}(\alpha(x, y) \wedge \beta(y, z)) .
$$

This is a straightforward generalisation of the composition of standard relations. It correctly describes the capacity behaviour along network paths: If there are edges from $x$ to $y$ and from $y$ to $z$ with capacities $\alpha(x, y)$ and $\beta(y, z)$ then at most $\alpha(x, y) \wedge \beta(y, z)$ can be transported from $x$ to $z$ along the concatenation of these two edges. Hence $\alpha \beta$ describes the supremum of transport capacity over all two-edge paths from $x$ to $z$.

Since the supremum of a bounded subset of the real numbers always exists and is unique, the composition of two fuzzy relations is well defined even if one of the sets $X, Y, Z$ is infinite.

A standard relation $R \subseteq X \times Y$ is called univalent if it relates every element of $X$ to at most one element of $Y$. This is expressed algebraically by the condition $R^{\sharp} R \subseteq i d_{Y}$. The dual notion of injectivity is characterised by $R R^{\sharp} \subseteq i d_{X}$. As a generalisation we call a fuzzy relation univalent if $\alpha^{\sharp} \alpha \sqsubseteq i d_{Y}$ holds. If $\alpha$ satisfies the property $\alpha \alpha^{\sharp} \sqsubseteq i d_{X}$ it is called injective.

The composition of fuzzy relations distributes over join, i.e., $\alpha(\beta \sqcup \gamma)=$ $\alpha \beta \sqcup \alpha \gamma$ and $(\alpha \sqcup \beta) \gamma=\alpha \gamma \sqcup \beta \gamma$. In general composition does not distribute over meet; in this case only $(\alpha \sqcap \beta) \gamma \sqsubseteq \alpha \gamma \sqcap \beta \gamma$ and analogously $\alpha(\beta \sqcap \gamma) \sqsubseteq \alpha \beta \sqcap \alpha \gamma$ hold. However, for univalent $\alpha$ the equality $\alpha(\beta \sqcap \gamma)=\alpha \beta \sqcap \alpha \gamma$ and for injective $\gamma$ the equality $(\alpha \sqcap \beta) \gamma=\alpha \gamma \sqcap \beta \gamma$ hold.

Composition commutes with scalar multiplication, i.e., $k(\alpha \beta)=(k \alpha) \beta=$ $\alpha(k \beta)$. Finally, composition is contravariant w.r.t. converse, i.e., $(\alpha \beta)^{\sharp}=\beta^{\sharp} \alpha^{\sharp}$.

Definition 2.8 (Powers and Star). The $n$-th power $\alpha^{n}$ of a fuzzy endorelation $\alpha: X \leftrightarrow X$ is defined inductively by $\alpha^{0}=i d_{X}$ and $\alpha^{n+1}=\alpha \alpha^{n}$ for $n \in \mathbb{N}_{0}$. The reflexive and transitive closure $\alpha^{*}$ of a fuzzy endorelation is defined by $\alpha^{*}=\bigsqcup_{n \in \mathbb{N}_{0}} \alpha^{n}$.

An elementary argument shows the equality $\alpha^{*}=\bigsqcup_{0 \leq n<|X|} \alpha^{n}$ for every fuzzy endorelation $\alpha$ on a finite set $X$.

\subsection{Cardinality of Fuzzy Relations}

For a standard relation $R \subseteq X \times Y$ its cardinality $|R|$ is the number of pairs in $R$; it coincides with the sum $\sum_{(x, y) \in X \times Y} \alpha(x, y)$ where $\alpha$ is the Boolean fuzzy relation corresponding to $R$, i.e., $\alpha(x, y)=1 \Leftrightarrow(x, y) \in R$. This is generalised in the following definition due to [Kaw].

Definition 2.9 (Cardinality). The cardinality $|\alpha|$ of a fuzzy relation $\alpha: X \leftrightarrow$ $Y$ is defined by $|\alpha|=\sum_{(x, y) \in X \times Y} \alpha(x, y)$. 
The cardinality will allow an elegant description of total weights of subnetworks. E.g., the cardinality of the relation consisting of all outgoing edges of a node describes the sum of the weights of all edges leaving that node. This is used, e.g., in Definition 3.2 of flows.

Obvious properties of the cardinality are:

- $|\alpha| \geq 0$

- $|\alpha|=0 \Leftrightarrow \alpha=\mathbf{0}$

- $|\alpha|=\left|\alpha^{\sharp}\right|$

- Cardinality is an isotone function, i.e., $\alpha \sqsubseteq \beta$ implies $|\alpha| \leq|\beta|$.

The cardinality of a fuzzy relation could become infinite if one of the participating sets is infinite; in this paper we won't deal with such cases. If both $X$ and $Y$ are finite sets the cardinality of a fuzzy relation $\alpha: X \leftrightarrow Y$ is always a nonnegative real number bounded by $|X| \cdot|Y|$.

A fuzzy relation $\alpha: X \leftrightarrow Y$ is called normalised if $|\alpha| \leq 1$. This implies $|\beta| \leq 1$ for all fuzzy relations $\beta$ with $\beta \sqsubseteq \alpha$. We will make use of normalised fuzzy relations when we want to label edges with the cardinality of a fuzzy relation.

A connection between meet and cardinality is the equation

$$
|\alpha \sqcup \beta|=|\alpha|+|\beta|-|\alpha \sqcap \beta|
$$

for arbitrary fuzzy relations $\alpha, \beta: X \leftrightarrow Y$. In particular, it states $|\alpha \sqcup \beta|=$ $|\alpha|+|\beta|$ for disjoint fuzzy relations $\alpha$ and $\beta$.

For fuzzy relations $\alpha, \beta, \gamma$ with $\beta \sqsubseteq \alpha$ and $\gamma \sqsubseteq \alpha \ominus \beta$ the equality $|\gamma|+|\beta|=$ $|\gamma \oplus \beta|$ holds. If $\beta \sqsubseteq \alpha$ then $|\alpha \ominus \beta|=|\alpha| \ominus|\beta|$.

\subsection{Test Relations}

Definition 2.10 (Test Relation). A Boolean subrelation of $i d_{X}$ is called a test relation on $X$.

A test relation $\tau$ corresponds in a natural way to the subset $\{x: \tau(x, x)=1\}$ of $X$; conversely, every subset $T$ of $X$ can be represented by the test relation $\tau$ with $\tau(x, x)=1 \Leftrightarrow x \in T$.

Hence the test relations form a Boolean subalgebra of $\operatorname{Rel}(X, X)$ with $\tau \sqcup \sigma$ being the join and $\tau \sqcap \sigma=\tau \sigma$ being the meet.

Consider a general fuzzy relation $\alpha$ and a test relation $\tau$. Then, by isotony of multiplication, $\tau \alpha \sqsubseteq i d \alpha=\alpha$. So $\tau \alpha$ is a part of $\alpha$; it omits all edges of $\alpha$ that do not start in a node in $\tau$. This models enforcing the precondition $\tau$. Similarly, $\alpha \tau$ removes all edges of $\alpha$ that do not end in a node in $\tau$; it enforces $\tau$ as a postcondition.

Combining this with the cardinality operator we can express the input or output capacity of a set of nodes characterised by $\tau$ compactly as $|\alpha \tau|$ and $|\tau \alpha|$, respectively.

The following property will be used several times: 
Lemma 2.11. Assume $\tau, \sigma, \alpha, \beta, \gamma: X \leftrightarrow X$ such that $\tau, \sigma$ are test relations with $\tau \sigma=\mathbf{0}$ and $\gamma \sqsubseteq \tau \alpha$ and $\gamma \sqsubseteq \sigma \beta$.

(a) $\gamma=\mathbf{0}$

(b) $|\tau \alpha \sqcup \sigma \beta|=|\tau \alpha|+|\sigma \beta|$.

Proof. Part (a) follows from the general theory of semirings (see Lemma A.4 in the Appendix). Part (b) holds by Equation (1), since by Part (a) $\tau \alpha \sqcap \sigma \beta=\mathbf{0}$.

Part (a) means that edge sets with disjoint sets of starting nodes are disjoint as well (remember that the composition of test relations is their conjunction).

To avoid excessive notation, in the remainder we will write $\tau$ both for a subset $\tau \subseteq X$ and its characterising test relation. The complement of $\tau$ relative to $X / i d_{X}$ is denoted by $\tau^{c}$.

Moreover, lower case latin letters will be used to denote elements $x \in X$ and the corresponding test relations characterising the singleton sets $\{x\}$. These relations are also called point relations.

Every test relation can be written as the join of suitable point relations, i.e., $U=\bigsqcup_{u \in U} u$ where the join ranges over point relations $u$. In the finite case this can be used to show properties of test relations via structural induction.

Using point relations we can also describe single graph edges. Given points $x, y$ and a weight $k \in[0,1]$, the fuzzy relation $k(x \nabla y)$ describes a graph connecting just $x$ and $y$ with edge weight $k$ : the test relations $x$ and $y$ reduce the universal relation $\nabla$ to the relation consisting only of the edge from $x$ to $y$ with weight 1 , and the weighting is done by scaling with the factor $k$.

\section{Flows in Networks}

Flows in networks were already treated in an algebraic manner in [Kaw] and [Glï]. We give a brief summary of the ideas from there relevant to the present paper. For the sake of well-definedness of cardinality let from now on all node sets be finite.

\subsection{Networks and Flows}

Definition 3.1 (Networks). For a set $X$ of nodes, a fuzzy endorelation $\alpha$ : $X \leftrightarrow X$ is also called a pseudo-network. For $x, y \in X$ we call $\alpha(x, y)$ the $c a$ pacity of $(x, y)$. If additionally $\alpha \sqcap \alpha^{\sharp}=\mathbf{0}$ then $N$ is called a network. An $s$-t(pseudo-)network is a triple $N=(\alpha: X \leftrightarrow X, s, t)$ consisting of a (pseudo-)network $\alpha$ and two distinct elements of $X$, namely $s$ (the source) and $t$ (the sink) of $N$.

Contrary to [Kaw] we admit input edges for the source and output edges for the sink, i.e., we give up the requirements $\alpha s=\mathbf{0}$ and $t \alpha=\mathbf{0}$, to obtain a more general concept. 
Our definition of a pseudo-network corresponds to that of a network as given for example in [Jun], Chapter 6, or [AMO], Chapter 1, or [GTT], except that there the capacities can have arbitrary values in $\mathbb{R}_{+}$.

Our network requirement that excludes antiparallel edges between two nodes by the condition $\alpha \sqcap \alpha^{\sharp}=0_{X X}$ may look too strong. But if we are given a fuzzy relation $\alpha: X \leftrightarrow X$ not fulfilling this condition we can construct a network $\hat{N}=$ $(\hat{\alpha}: \hat{X} \leftrightarrow \hat{X}, s, t)$ as follows: for all pairs $(x, y) \in X \times Y$ with $\alpha(x, y) \sqcap \alpha^{\sharp}(x, y) \neq 0$ we introduce two additional nodes $x^{\prime}$ and $y^{\prime}$ and set $\hat{\alpha}(x, y)=\hat{\alpha}(y, x)=\hat{\alpha}\left(x^{\prime}, y^{\prime}\right)$ $=\hat{\alpha}\left(y^{\prime}, x^{\prime}\right)=0, \hat{\alpha}\left(x, x^{\prime}\right)=\hat{\alpha}\left(x^{\prime}, y\right)=\alpha(x, y), \hat{\alpha}\left(y, y^{\prime}\right)=\hat{\alpha}\left(y^{\prime}, x\right)=\alpha(y, x)$ and $\hat{\alpha}\left(x^{\prime}, x\right)=\hat{\alpha}\left(y, x^{\prime}\right)=\hat{\alpha}\left(y^{\prime}, y\right)=\hat{\alpha}\left(x, y^{\prime}\right)=0$.

This strategy turns a pair of antiparallel edges into a bypassing device:
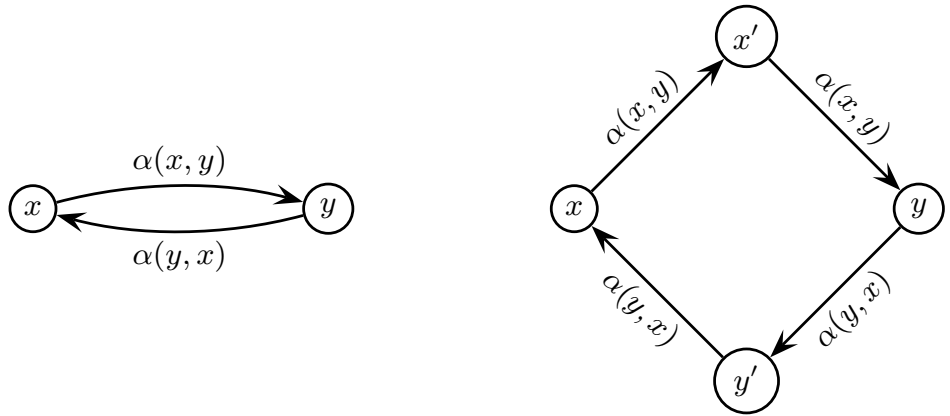

Fig. 1. Bypassing device

In a quite natural way the definition of a network flow (cf. again the standards [Jun] and $[\mathrm{AMO}])$ is translated into the language of fuzzy relations:

Definition 3.2 (Flow). A flow $\varphi$ in a pseudo-network $\alpha: X \leftrightarrow X$ is a fuzzy endorelation $\varphi: X \leftrightarrow X$ such that $\varphi \sqsubseteq \alpha$ and $|\tau \varphi|=|\varphi \tau|$ for all test relations $\tau: X \leftrightarrow X$ on $X$. A flow $\varphi$ in an $s$-t-(pseudo-)network $(\alpha: X \leftrightarrow X, s, t)$ is a fuzzy endorelation on $X$ with $\varphi \sqsubseteq \alpha$ and $|\tau \varphi|=|\varphi \tau|$ for all test relations $\tau$ with $\tau \sqsubseteq X \backslash\{s, t\}$.

The first part of this definition is the capacity constraint: the flow along an edge can be at most as high as allowed by the capacity of that edge. The second part corresponds to the flow conservation, commonly written as

$$
\sum_{v \in V} \varphi(v, u)=\sum_{v \in V} \varphi(u, v) \forall u \in X \backslash\{s, t\},
$$

see, e.g., [Jun], p.147 (with a slightly different notation). Our version of flow conservation seems to be stronger than $(*)$, but the equivalence of the two formulations can be shown by induction over the size of the tests (cf. [Glü], Section 6.2). There is always at least the trivial flow $\mathbf{0}$ in any network $N=(\alpha: X \leftrightarrow X, s, t)$. 
Because of flow conservation, in bypassing devices as in Fig. 1 there is a one-to-one correspondence between flows in the original pseudo-network and the modified network.

\subsection{The Max-Flow Min-Cut Theorem}

A flow can be seen as a possibility of transporting a certain amount from $s$ to $t$. So a flow is the more valuable the more it transports from $s$ to $t$. This motivates the next definition:

Definition 3.3 (Value of a Flow). The value of a flow $\varphi$ in a network $N=$ $(\alpha: X \leftrightarrow X, s, t)$ is defined as $\operatorname{val}(\varphi)=|s \varphi|-|\varphi s|$.

The value measures how much $s$ "generates" in addition to its own input $|\varphi s|$. In Prop. 6.1 of [Glü] it has been shown (in a slightly different notation) that the equality $\operatorname{val}(\varphi)=|s \varphi|-|\varphi s|=|\varphi t|-|t \varphi|$ holds. Note that the value of a flow can even be a negative number as shown in the following example:

Example 3.4.

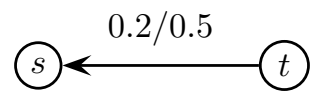

In this network $\alpha$ consists of the single edge $(t, s)$ with capacity 0.5 , and the depicted flow $\varphi$ is given by $\varphi(t, s)=0.2$. So we have $\operatorname{val}(\varphi)=|s \varphi|-|\varphi s|=$ $0-0.2=-0.2$. However, our main aim will be to send flow from $s$ to $t$.

Definition 3.5 (Residual Network). For a flow $\varphi$ on an $s$-t-network $N=$ $(\alpha: X \leftrightarrow X, s, t)$ we define the residual network $N_{\alpha}$ of $N$ with respect to $\alpha$ as the pseudo-network $N_{\alpha}=\left(\varphi_{\alpha}: X \leftrightarrow X, s, t\right)$ with $\varphi_{\alpha}=(\alpha \ominus \varphi) \sqcup \varphi^{\sharp}$.

That means, a flow $\varphi$ over an edge $(x, y)$ with capacity $\alpha(x, y)$ causes two edges in the residual network: one edge $(x, y)$ with capacity $\alpha(x, y)-\varphi(x, y)$ (note that $\varphi \sqsubseteq \alpha)$ and one edge $(y, x)$ in the opposite direction with capacity $\varphi(x, y)$. The intuitive meaning is that one can send along the edge $(x, y)$ an additional flow amount of at most $\alpha(x, y)-\varphi(x, y)$ and the flow along the edge $(x, y)$ can be decreased by an amount of at most $\varphi(x, y)$, which corresponds to increasing the flow over the reverse edge $(y, x)$ by exactly the same amount. In this context the requirement $\alpha \sqcap \alpha^{\sharp}=\mathbf{0}$ is indispensable.

An important concept for reasoning about flows is that of a cut.

Definition 3.6 (Cut). A cut in an $s$-t-network $N=(\alpha: X \leftrightarrow X, s, t)$ is a test relation $\tau$ on $X$ with the property $s \sqsubseteq \tau \sqsubseteq t^{c}$. Thus a cut corresponds to a subset of $X$ containing $s$ but not $t$, a definition consistent with the usual one. The capacity $c(\tau)$ of a cut $\tau$ is given by $c(\tau)=\left|\tau \alpha \tau^{c}\right|$. It describes the maximal flow amount which can be sent out from nodes in $\tau$ to nodes in $\tau^{c}$. A cut $\tau$ is saturated by a flow $\varphi$ if $\operatorname{val}(\varphi)=c(\tau)$. 
Intuitively it is obvious that the value of all flows can not exceed the capacity of a cut, because a cut forms a border between the source and the sink. This basic fact is proved both in [Kaw] and [Glü].

Given a network $N=(\alpha: X \leftrightarrow X, s, t)$, our main aim is to determine a flow of maximal value, i.e., a flow $\varphi$ with the property $\operatorname{val}(\varphi) \geq \operatorname{val}(\psi)$ for all flows $\psi$ on $N$. An important criterion for the maximality of a flow is the so-called Max-Flow Min-Cut Theorem:

Theorem 3.7 (Max-Flow Min-Cut Theorem). Let $N=(\alpha: X \leftrightarrow X, s, t)$ be network and $\varphi$ a flow on $N$. Then the following properties are equivalent:

(a) $\varphi$ is maximal.

(b) $t \sqcap s \varphi_{\alpha}^{*}=\mathbf{0}$, or equivalently $\left|s \varphi_{\alpha}^{*} t\right|=0$.

(c) There exists a cut $\tau$ that is saturated by $\varphi$.

Intuitively part (b) means that it is impossible to send flow from $s$ to $t$ in the residual network of a maximal flow. Part (c) states that the value of a maximal flow equals the capacity of a certain cut. Because the value of a flow is never larger than the capacity of a cut, this cut must be minimal (hence the name Max-Flow Min-Cut Theorem). Such a cut $\tau$ is saturated by a maximal flow $\varphi$; in this case $\tau \alpha \tau^{c}=\tau \varphi \tau^{c}$ holds. This means that every edge of $\alpha$ leading from $\tau$ into its complement $\tau^{c}$ carries the maximally possible amount of flow.

\section{Networks with Lower Bounds}

\subsection{Definitions and Background}

Definition 4.1 (Networks With Lower Bounds). A pseudo-network with lower bounds over a node set $X$ is a pair $N=(\alpha: X \leftrightarrow X, \beta: X \leftrightarrow X)$ with $\beta \sqsubseteq \alpha$, where $\alpha$ and $\beta$ are called the upper and lower bound. If additionally $\alpha \sqcap \alpha^{\sharp}=\mathbf{0}$ then $N$ is called a network with lower bounds.

Our definition of a (pseudo-)network with lower bounds corresponds to the common one in an analogous way as that of an $s$-t-(pseudo-)network. The network condition implies $\alpha \sqcap \beta^{\sharp}=\mathbf{0}, \alpha^{\sharp} \sqcap \beta=\mathbf{0}$ and $\beta \sqcap \beta^{\sharp}=\mathbf{0}$ (note that in the semiring of fuzzy endorelations on $X$ the zero $\mathbf{0}$ is meet-irreducible).

We represent networks with lower bounds in a graphical way analogous to the common one for graphs. We associate the elements of the basic underlying set with the nodes of a graph and label its edges with $[\beta, \alpha]$ where $\beta$ and $\alpha$ denote the lower and upper capacity bound of the edge, resp. Edges $(x, y)$ with $\alpha(x, y)=0$ (and hence $\beta(x, y)=0$ ) are omitted in the depiction. 
Example 4.2.

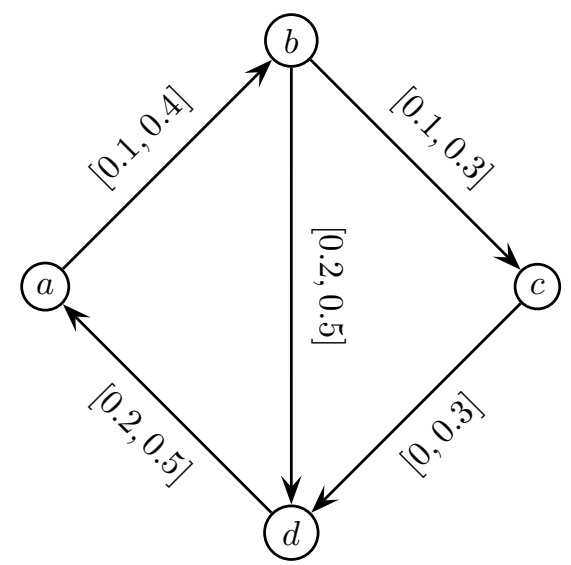

In this example we have $X=\{a, b, c, d\}$. For the upper bound we have, e.g., $\alpha(b, d)=0.5$ or $\alpha(b, c)=0.3$ and for the lower bound $\beta(a, b)=0.1$ or $\beta(d, a)=$ 0.2 . For pairs $(x, y) \in X \times Y$ without arcs in the depiction we have $\alpha(x, y)=$ $\beta(x, y)=0$, so for example $\alpha(a, c)=\beta(a, c)=0$ or $\alpha(a, d)=\beta(a, d)=0$.

\subsection{Circulations in Networks with Lower Bounds}

Definition 4.3 (Circulation). A fuzzy relation $\gamma$ in a pseudo-network $N=$ $(\alpha: X \leftrightarrow X, \beta: X \leftrightarrow X)$ with lower bounds is called a circulation if it is a flow in the network $\alpha$ and satisfies the additional capacity constraint $\beta \sqsubseteq \gamma$.

A circulation in a network with lower bounds need not always exist. A simple counterexample is given here:

Example 4.4.

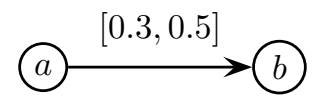

Every fuzzy endorelation $\gamma$ on $X$ fulfilling the capacity constraint and flow conservation needs to satisfy $0.3 \leq|\gamma b| \leq 0.5$ and $|b \gamma|=0$ and hence cannot satisfy the requirement $|\gamma b|=|b \gamma|$.

Contrary to this, our network with lower bounds from Example 4.2 admits a circulation as one can see here: 


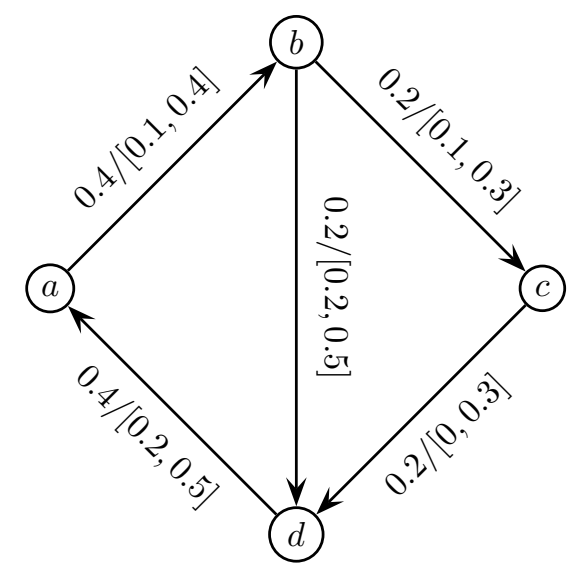

An edge $(x, y)$ carries the information $\gamma(x, y) /[\beta(x, y), \alpha(x, y)]$.

\subsection{Extending Pseudo-Networks}

In the next section we will need to add nodes to a network. To this end we introduce some notation.

Definition 4.5 (Embedding). For a fuzzy endorelation $\alpha: X \leftrightarrow X$ on $X$ and a superset $\hat{X}$ of $X$, the embedding $\lceil\alpha\rceil$ of $\alpha$ into $\hat{X}$ is $\lceil\alpha\rceil: \hat{X} \leftrightarrow \hat{X}$, given by $\lceil\alpha\rceil(x, y)=\alpha(x, y)$ for all $(x, y) \in X \times X$ and $\lceil\alpha\rceil(x, y)=0$ otherwise.

This means that the nodes in $\hat{X} \backslash X$ are added as isolated nodes to the graph described by $\alpha$.

The embedding of fuzzy relations distributes over join, meet, truncating sum, truncating difference and composition, i.e., $\lceil\alpha \circ \beta\rceil=\lceil\alpha\rceil \circ\lceil\beta\rceil$ for $\circ \in$ $\{\sqcup, \sqcap, \oplus, \ominus, \cdot\}$. In particular we have $\lceil\alpha\rceil=\lceil i d\rceil\lceil\alpha\rceil=\lceil\alpha\rceil\lceil i d\rceil$. Embedding also commutes with converse, i.e., $\left\lceil\alpha^{\sharp}\right\rceil=\lceil\alpha\rceil^{\sharp}$. Moreover, it is order-preserving: $\alpha \sqsubseteq \beta$ implies $\lceil\alpha\rceil \sqsubseteq\lceil\beta\rceil$. The embedding $\lceil\tau\rceil$ of a test relation $\tau$ on $X$ is a test relation on $\hat{X}$. Finally, embedding preserves cardinality: $|\lceil\alpha\rceil|=|\alpha|$.

The dual operation to embedding is projection:

Definition 4.6 (Projection). For a fuzzy endorelation $\alpha: \hat{X} \leftrightarrow \hat{X}$ its projection $\lfloor\alpha\rfloor: X \leftrightarrow X$ to a subset $X \subseteq \hat{X}$ is given by $\lfloor\alpha\rfloor(x, y)=\alpha(x, y)$ for all $x, y \in X$.

Projection has algebraic properties similar to the ones of embedding. It is order preserving and commutes with converse and distributes over join, meet, truncating sum and truncating difference, but in general not over composition. The projection $\lfloor\hat{\tau}\rfloor$ of a test relation $\hat{\tau}$ on $\hat{X}$ is a test relation on $X$. Finally, projection partially preserves cardinality: for $\sigma, \tau \subseteq X$ one has $|\lfloor\sigma \hat{\alpha} \tau\rfloor|=|\sigma \hat{\alpha} \tau|$. 


\subsection{Existence of Circulations}

Let $N=(\alpha: X \leftrightarrow X, \beta: X \leftrightarrow X)$ be a network with lower bounds with a normalised fuzzy relation $\alpha$ as upper bound (recall that this means $|\alpha| \leq 1$ ). This can be achieved by a suitable scaling of the upper bound. Note that if $\alpha$ is normalised then $\beta$ is, too. Because of $x \beta \sqsubseteq \beta$ for an arbitrary point relation $x$ we have $|x \beta| \leq 1$ and analogously $|\beta x| \leq 1$.

Now our intention is to develop in an algebraic way an algorithm that determines whether a network with lower bounds admits a circulation and, if so, computes one. This will be done using a maximal flow in an $s$-t-network derived from the original network. The construction we will use is well known in the literature, cf. [Jun], Section 10.2. The advantage of our approach is that we avoid big indexed sums by calculating with test relations and cardinalities.

To this end we define the $s$-t-pseudo-network $\hat{N}=(\hat{\alpha}: \hat{X} \leftrightarrow \hat{X}, s, t)$ as follows: we choose two new nodes $s, t \notin X$ and set $\hat{X}=X \dot{\cup}\{s\} \dot{\cup}\{t\}$. For the associated point relations $s$ and $t$ on $\hat{X}$ this means $s \sqcap t=\mathbf{0}$ and $(s \sqcup t) \sqcap x=\mathbf{0}$ for all point relations $x \sqsubseteq X$.

As abbreviations for $\bar{\nabla}_{\hat{X} \hat{X}}, \mathbf{0}_{\hat{X} \hat{X}}$ and $\nabla_{X X}, \mathbf{0}_{X X}$ we use $\hat{\nabla}, \hat{\mathbf{0}}$ and $\nabla, \mathbf{0}$, resp. With this notation the equalities $\lfloor\hat{\nabla}\rfloor=\nabla$ and $\lfloor\hat{\mathbf{0}}\rfloor=\mathbf{0}$ hold.

We define the capacity constraint $\hat{\alpha}$ of $\hat{N}$ by $\hat{\alpha}=\hat{\alpha}_{1} \sqcup \hat{\alpha}_{2} \sqcup \hat{\alpha}_{3}$ with three fuzzy endorelations $\hat{\alpha}_{1}, \hat{\alpha}_{2}$ and $\hat{\alpha}_{3}$ on $\hat{X}$. First, $\hat{\alpha}_{1}=\bigsqcup_{x \in X}|\beta x| s \hat{\nabla}\lceil x\rceil$. Intuitively this means that we have an edge from $s$ to every point $x$ of $X$ having as capacity the sum of the lower bounds of all edges entering $x . \hat{\alpha}_{3}$ is defined in a similar manner, namely as $\hat{\alpha}_{3}=\bigsqcup_{x \in X}|x \beta|\lceil x\rceil \hat{\nabla} t$. This introduces an edge from every point $x$ of $X$ to $t$ having as capacity the sum of the lower bounds of all edges leaving $x$. Finally, $\hat{\alpha}_{2}$ is defined as $\hat{\alpha}_{2}=\lceil\alpha \ominus \beta\rceil$. This means that every edge in $N$ becomes an edge in $\hat{N}$ having as capacity the difference between its upper and lower bounds (remember that $\beta \sqsubseteq \alpha$ ). The relation $\hat{\alpha}$ is well defined because all terms of the form $|x \beta|$ and $|\beta x|$ are guaranteed not to be larger than one.

Example 4.\%. If we apply this construction to the network with lower bounds shown in Example 4.2 we obtain the following network:

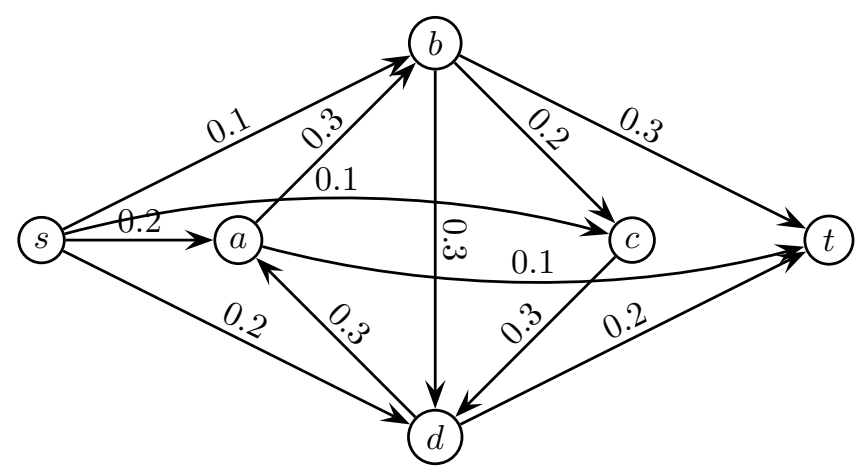

The edge from $c$ to $t$ has not been forgotten, but we have $\hat{\alpha}(c, t)=0$ and according to our conventions we omit the arc corresponding to this pair. 
Lemma 4.8. $\hat{N}$ is an s-t-network.

Proof. We have to show $\hat{\alpha} \sqcap \hat{\alpha}^{\sharp}=\hat{\mathbf{0}}$. As a preparation we calculate

$$
\begin{aligned}
&= \hat{\alpha} \sqcap \hat{\alpha}^{\sharp} \\
&\{[\text { definition and rules for converse }]\} \\
&\left(\hat{\alpha}_{1} \sqcup \hat{\alpha}_{2} \sqcup \hat{\alpha}_{3}\right) \sqcap\left(\hat{\alpha}_{1}^{\sharp} \sqcup \hat{\alpha}_{2}^{\sharp} \sqcup \hat{\alpha}_{3}^{\sharp}\right) \\
&=\{[\text { distributivity }]\} \\
& \bigsqcup_{i=1}^{3} \sqcup_{j=1}^{3}\left(\hat{\alpha}_{i} \sqcap \hat{\alpha}_{j}^{\sharp}\right) .
\end{aligned}
$$

If we succeed in showing that all meets inside the two big joins become $\hat{\mathbf{0}}$ we are done.

For the case $i=j=1$ we have

$$
\begin{aligned}
= & \hat{\alpha}_{1}^{\sharp}\{[\text { converse distributes over join }\}\} \\
= & \bigsqcup_{x \sqsubseteq X}|\beta x|\lceil x\rceil^{\sharp} \hat{\nabla}^{\sharp} s^{\sharp} \\
& \{\text { universal and test relations are their own converses }\}\} \\
= & \bigsqcup_{x \sqsubseteq X}|\beta x|\lceil x\rceil \hat{\nabla} s \\
& \left.\bigsqcup_{x \sqsubseteq X}|\beta x|\lceil\text { rules for embedding }\}\right\}
\end{aligned}
$$

Hence

$$
\begin{aligned}
& \hat{\alpha}_{1} \sqcap \hat{\alpha}_{1}^{\sharp} \\
& =\{\text { distributivity }\} \\
& \bigsqcup_{x, y \in X}|\beta x| s \hat{\nabla}\lceil x\rceil \sqcap|\beta y|\lceil i d\rceil\lceil y\rceil \hat{\nabla} s \\
& \leq \quad\{\text { isotony of meet and join }\} \\
& \bigsqcup_{x, y \in X} s \hat{\nabla}\lceil x\rceil \sqcap\lceil i d\rceil\lceil y\rceil \hat{\nabla} s \\
& =\quad\{\text { by } s\lceil i d\rceil=\hat{\mathbf{0}} \text { and Lemma 2.11(a) }\} \\
& \bigsqcup_{x, y \in X} \hat{\mathbf{0}} \\
& =\{[\text { lattice algebra }\}
\end{aligned}
$$

In the case $i=3$ and $j=1$ we have to consider $\hat{\alpha}_{3} \sqcap \hat{\alpha}_{1}^{\sharp}$. Calculations similar to those above lead to $\hat{\alpha}_{3} \sqcap \hat{\alpha}_{1}^{\sharp}=\bigsqcup_{x, y \in X}|x \beta|\lceil x\rceil \hat{\nabla} t \sqcap|\beta y|\lceil y\rceil \hat{\nabla} s$. Because of $s t=\hat{\mathbf{0}}$ we obtain again $\hat{\mathbf{0}}$ by Lemma 2.11(a).

The other cases, except $i=j=2$, can be treated in a similar manner (note that $\left.\hat{\alpha}_{2}=\lceil i d\rceil \hat{\alpha}_{2}=\hat{\alpha}_{2}\lceil i d\rceil\right)$. For $i=j=2$ we calculate $\hat{\alpha}_{2} \sqcap \hat{\alpha}_{2}^{\sharp}=$ $\lceil\alpha \ominus \beta\rceil \sqcap\lceil\alpha \ominus \beta\rceil^{\sharp} \sqsubseteq\lceil\alpha\rceil \sqcap\lceil\alpha\rceil^{\sharp}=\left\lceil\alpha \sqcap \alpha^{\sharp}\right\rceil$, and this equals $\hat{\mathbf{0}}$ by the requirement that $N$ be a network with lower bounds.

Lemma 4.9. A maximal flow on $\hat{N}$ with value $|\beta|$ saturates the cuts $s$ and $t^{c}$.

Proof. First we show that the capacities of the cuts $s$ and $t^{c}$ in $\hat{N}$ both equal $|\beta|$. We have 


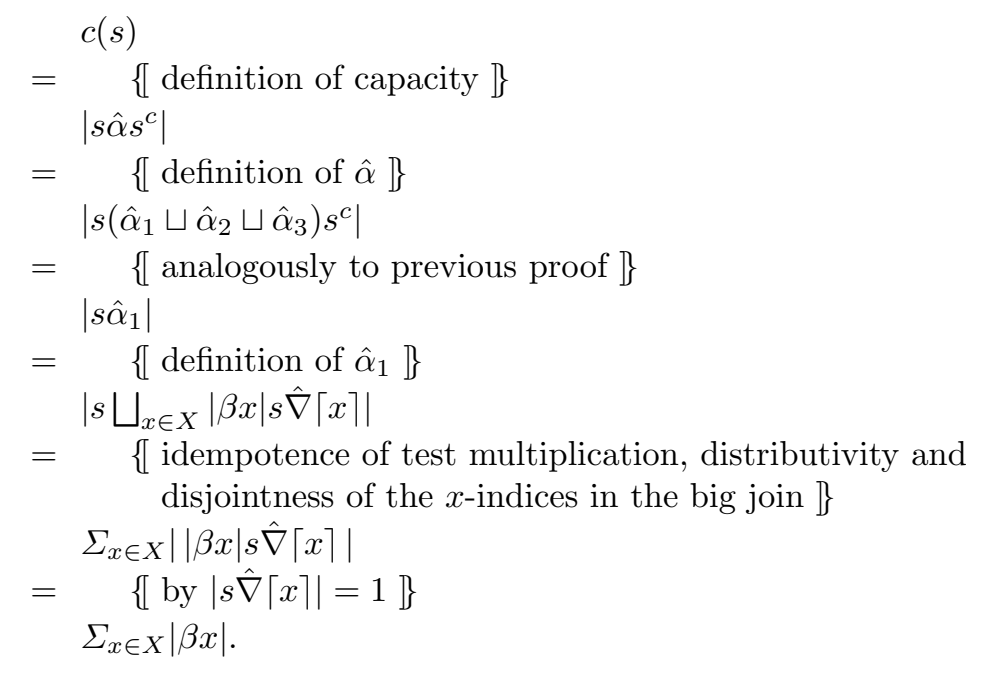

Rewriting the join in the opposite direction we obtain $\Sigma_{x \in X}|\beta x|=\| \dot{ப}_{x \in X} \beta x \mid$ $=\left|\beta i d_{X}\right|=|\beta|$.

In the same manner one can show $c\left(t^{c}\right)=|\beta|$. According to the assertions of the Max-Flow Min-Cut Theorem a maximal flow with value $|\beta|$ has to saturate both cuts.

Theorem 4.10. There is a circulation on $N$ iff the value of a maximal flow on $\hat{N}$ is exactly $|\beta|$.

Proof. $(\Leftarrow)$ Let $\hat{\varphi}$ be a flow on $\hat{N}$ with value $\operatorname{val}(\hat{\varphi})=|\beta|$. Then we define a fuzzy relation $\gamma: X \leftrightarrow X$ by $\gamma=\lfloor\hat{\varphi}\rfloor \oplus \beta$. Because $\hat{\varphi}$ is a flow on $\hat{N}$ it has to respect the capacity constraint, which implies $\gamma \sqsubseteq\lfloor\hat{\alpha}\rfloor=\hat{\alpha} \ominus \beta$ and hence $\gamma \sqsubseteq \hat{\alpha}$. $\beta \sqsubseteq \gamma$ is clear because of the definition of $\gamma$. If we succeed in showing that $\gamma$ satisfies flow conservation on $N$ we are done.

Consider an arbitrary test relation $\tau: X \leftrightarrow X$. Because of the flow properties of $\hat{\varphi}$ we have

$$
|\lceil\tau\rceil \hat{\varphi}|=|\hat{\varphi}\lceil\tau\rceil| \text {. }
$$

We introduce a test relation $X$ on $\hat{X}$ by $X=\left\lceil i d_{X}\right\rceil=(s \sqcup t)^{c}$. Note that $i d_{\hat{X}}=X \sqcup s \sqcup t$.

Now we reason as follows:

$$
\begin{array}{ll} 
& |\lceil\tau\rceil \hat{\varphi}|=|\hat{\varphi}\lceil\tau\rceil| \\
\Leftrightarrow & \{\text { identity }\} \\
& \left|\lceil\tau\rceil \hat{\varphi} i d_{\hat{X}}\right|=\left|i d_{\hat{X}} \hat{\varphi}\lceil\tau\rceil\right| \\
\Leftrightarrow & \{\mid \text { above remark }\} \\
& |\lceil\tau\rceil \hat{\varphi}(X \sqcup s \sqcup t)|=|(X \sqcup s \sqcup t) \hat{\varphi}\lceil\tau\rceil| \\
\Leftrightarrow & \{\mid X, s, t \text { disjoint and Lemma } 2.11(\mathrm{~b})\} \\
& |\lceil\tau\rceil \hat{\varphi} X|+|\lceil\tau\rceil \hat{\varphi} s|+|\lceil\tau\rceil \hat{\varphi} t|=|X \hat{\varphi}\lceil\tau\rceil|+|s \hat{\varphi}\lceil\tau\rceil|+|t \hat{\varphi}\lceil\tau\rceil| \\
\Rightarrow & \{\mid \hat{\alpha} s=\hat{\mathbf{0}} \wedge \hat{\varphi} \sqsubseteq \hat{\alpha} \Rightarrow \hat{\varphi} s=\hat{\mathbf{0}}, \text { analogously } t \hat{\varphi}=\hat{\mathbf{0}}\}
\end{array}
$$




$$
\begin{array}{ccc} 
& |\lceil\tau\rceil \hat{\varphi} X|+|\lceil\tau\rceil \hat{\varphi} t|=|X \hat{\varphi}\lceil\tau\rceil|+|s \hat{\varphi}\lceil\tau\rceil| \\
\Rightarrow & \{\hat{\varphi} \text { maximal with value }|\beta|, \text { Lemma 4.9 }\} \\
& |\lceil\tau\rceil \hat{\varphi} X|+|\lceil\tau\rceil \hat{\alpha} t|=|X \hat{\varphi}\lceil\tau\rceil|+|s \hat{\alpha}\lceil\tau\rceil| \\
\Rightarrow & \{\{\text { definition of } \hat{\alpha}\} \\
& |\lceil\tau\rceil \hat{\varphi} X|+|\tau \beta|=|X \hat{\varphi}\lceil\tau\rceil|+|\beta \tau| \\
\Rightarrow & \{\text { definition of } X\} \\
& |\tau\lfloor\hat{\varphi}\rfloor|+|\tau \beta|=|\lfloor\hat{\varphi}\rfloor \tau|+|\beta \tau| \\
\Rightarrow & \{\lfloor\hat{\varphi}\rfloor \sqsubseteq\lfloor\hat{\alpha}\rfloor=\alpha \ominus \beta, \text { rules of cardinality }\} \\
& |\tau(\lfloor\hat{\varphi}\rfloor \oplus \beta)|=|(\lfloor\hat{\varphi}\rfloor \oplus \beta) \tau| .
\end{array}
$$

$(\Rightarrow)$ Let $\gamma$ be a circulation on $N$. Then we construct a fuzzy relation $\hat{\varphi}: \hat{X} \leftrightarrow \hat{X}$ by $\hat{\varphi}=s \hat{\alpha} \sqcup\lceil\gamma \ominus \beta\rceil \sqcup \hat{\alpha} t$. Because $\gamma$ satisfies the capacity constraint and because of the construction of $\hat{\varphi}$ the capacity constraint on $\hat{N}$ is satisfied by $\hat{\varphi}$. The value of the thus constructed flow is $|s \hat{\alpha}|=|\beta|$, and it is maximal because it saturates the cut $s$. Flow conservation can be shown similarly as above.

\subsection{Algorithmic Aspects}

We wouldn't be computer scientists if we didn't give an algorithm for computing a circulation in a network with lower bounds. The construction from the proof of Theorem 4.10 can immediately be used to construct from an algorithm for the computation of a maximal flow in an $s$-t-network an algorithm for determining a circulation in a network with lower bounds:

(1) Determine the $s$-t-network $\hat{N}$ as described above.

(2) Determine a maximal flow $\varphi$ on $\hat{N}$.

(3) If $\operatorname{val}(\varphi) \neq \beta$ then no circulation in $N$ is possible; otherwise return $\gamma$ as described above.

An algorithm for computing a maximal flow in an s-t-network based on fuzzy relations has been derived in [Kaw].

Now we try out the above algorithm on our running example. E.g. by Kawahara's method one can find the following flow on the induced $s$-t-network: 


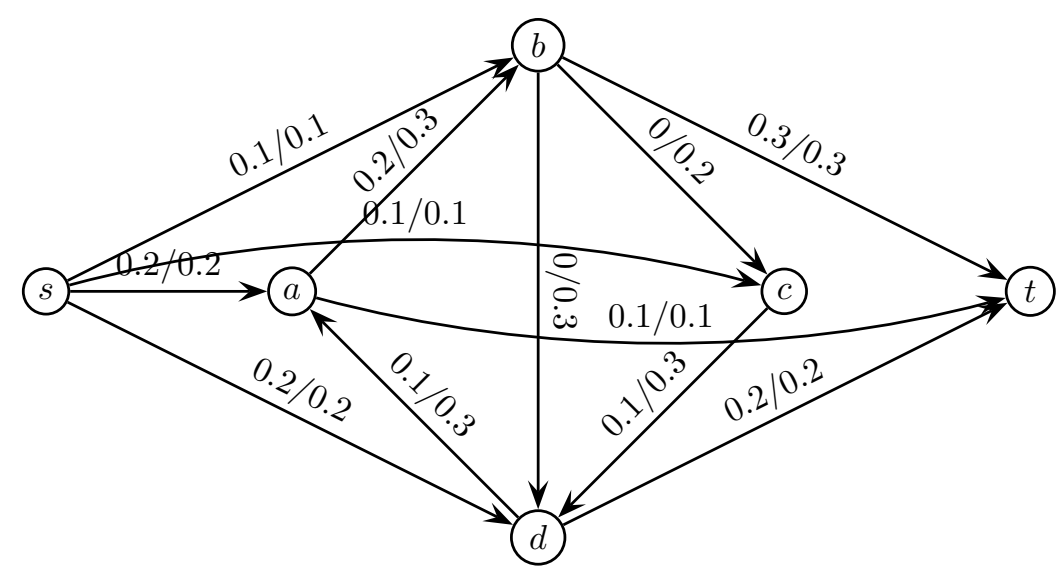

This flow is indeed maximal, because it saturates the cut $s$. Applying our algorithm we obtain the following circulation:

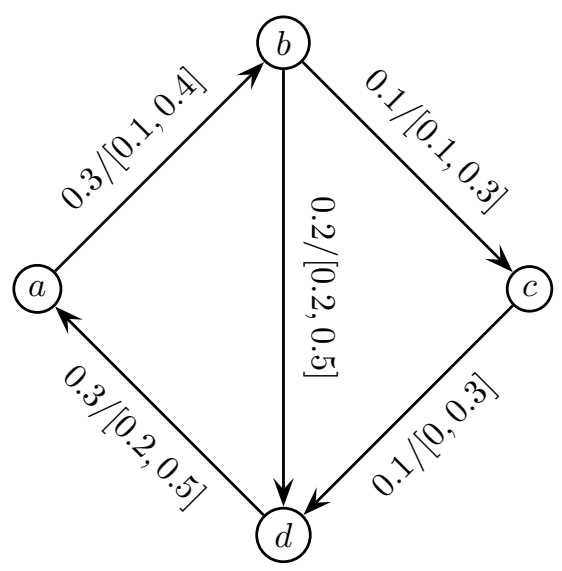

This circulation is different from the one already shown in the second picture of Example 4.4.

\subsection{Another Existence Criterion}

We will now derive a more elegant criterion for the existence of a flow in a network with lower bounds. We notice that there is a bijective mapping between tests on $N$ and cuts on $\hat{N}$ : for a test relation $\tau$ on $N$ we choose the cut $s \sqcup\lceil\tau\rceil$ as corresponding cut on $\hat{N}$; the converse direction is obvious.

For the capacity of such a cut $s \sqcup\lceil\tau\rceil$ we can calculate as follows (note that by construction $s^{c}=X \sqcup t, t^{c}=s \sqcup X, X^{c}=s \sqcup t$ and $\lceil\tau\rceil^{c}=\left\lceil\tau^{c}\right\rceil \sqcup s \sqcup t$ hold): 


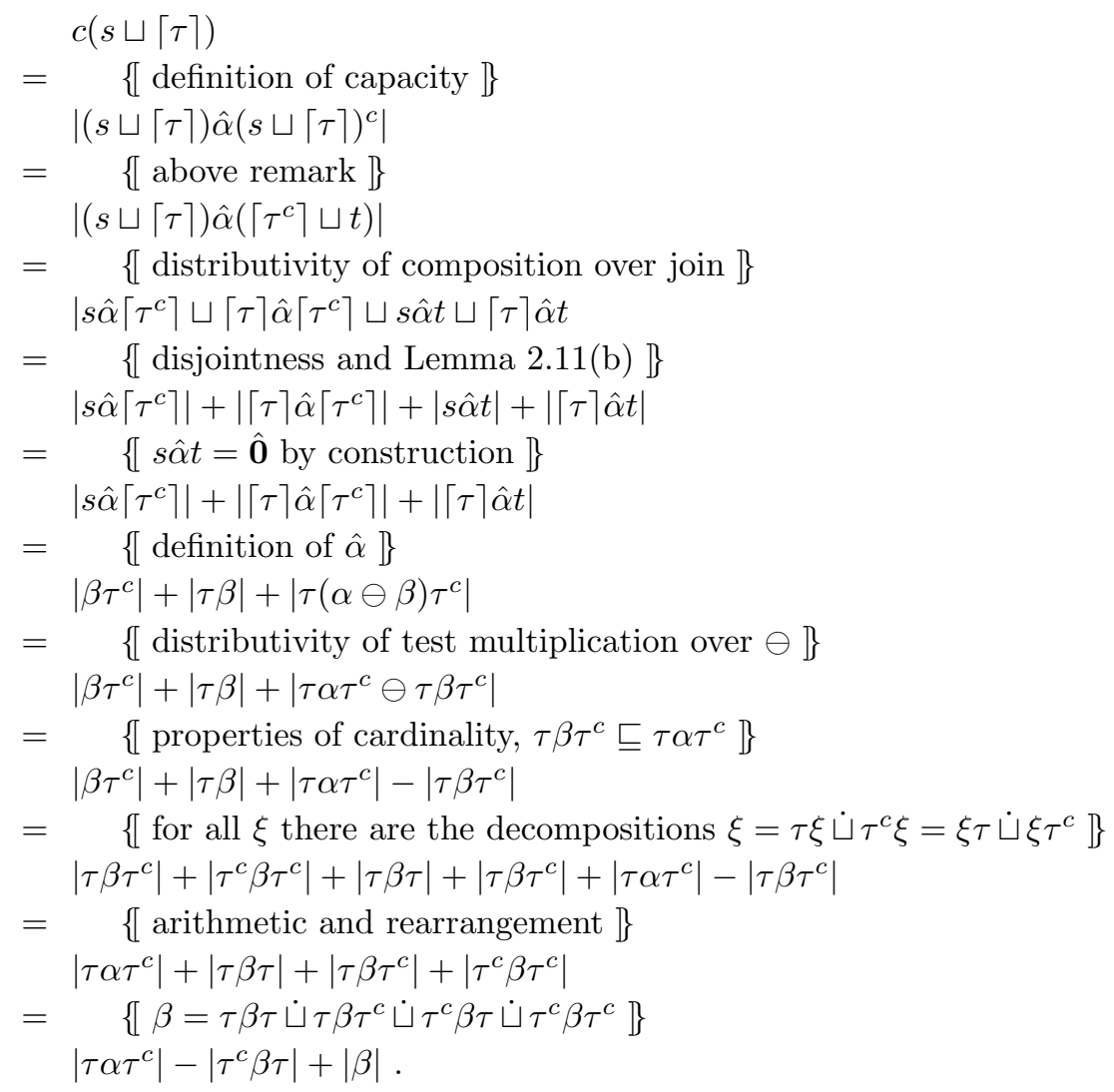

By the previous theorem $N$ admits a circulation iff the value of a maximal flow on $\hat{N}$ equals $|\beta|$, so according to the Max-Flow Min-Cut Theorem $\left|\tau \alpha \tau^{c}\right|-$ $\left|\tau^{c} \beta \tau\right|$ has to be $\geq 0$ for all test relations $\tau$ on $N$ iff $N$ admits a circulation. So we have proved the following theorem:

Theorem 4.11. A network with lower bounds $N=(\alpha: X \leftrightarrow X, \beta: X \leftrightarrow X)$ admits a circulation iff the condition $\left|\tau \alpha \tau^{c}\right| \geq\left|\tau^{c} \beta \tau\right|$ holds for all test relations $\tau$ on $X$.

This theorem is not very suitable for application in practice (the number of test relations is growing exponentially in the number of nodes!), but it can serve well in the theoretical investigation of networks with lower bounds.

\section{Conclusion and Future Work}

We have applied Kawahara's methods developed for flows in networks successfully to circulations in networks with lower bounds. As shown in [GlüI], it is not difficult to do the same for circulations in networks with additional imports as described in [Jun], Chapter 7. There a node can receive an additional amount of flow by the environment or flow can disappear from a node to the outside. 
In all cases the basic mathematical tools are fuzzy relations and their cardinality. As we detail in the Appendix, these form a semiring (and even a Kleene algebra) with tests. Since automated reasoning about Kleene algebras is on the rise (cf. [HS]), we expect the first automatically proved theorems in this area soon.

Another challenge in this area will be the description of min-cost flows (cf. [Jun], Chapter 10) with fuzzy relational methods. This will be a little bit more difficult, because in the problem of a min-cost flow the amount of flow is multiplied by a cost function on the edge it crosses. For this purpose we have first to define an operation for the edgewise multiplication of fuzzy relations and to explore its algebraic laws and its behavior in connection with the cardinality.

Acknowledgment We are grateful to Peter Höfner, Yasuo Kawahara, Walter Vogler and the anonymous referees for helpful comments and suggestions.

\section{A Appendix: Semirings and Kleene Algebras}

In this section we embed fuzzy relations into the more general mathematical framework of semirings and Kleene algebras. This allows re-use of many results from there and also automatic proofs of some relevant properties.

\section{A.1 Introduction}

Definition A.1 (Semiring). A semiring is a quintuple $(M,+, \cdot, 0,1)$ fulfilling the following properties:

- $(M,+, 0)$ is a commutative monoid.

- $(M, \cdot, 1)$ is a monoid.

- 0 is an annihilator with respect to ·, i.e.,

$$
\forall m \in M: m \cdot 0=0 \cdot m=0
$$

- distributes over +, i.e.,

$$
\forall a, b, c \in M: a \cdot(b+c)=(a \cdot b)+(a \cdot c),(a+b) \cdot c=(a \cdot c)+(b \cdot c)
$$

We call + and $\cdot$ addition and multiplication, resp.

Elements of a semiring can, e.g., be considered as modelling some sort of transition relation, such as the transition from one graph node to another along an edge. An element $x+y$ corresponds to a choice between $x$ and $y$, while $x \cdot y$ corresponds to the sequential composition of $x$ and $y$ in that order.

As one can see the fuzzy endorelations over a set $X$ form a semiring with join as addition, composition as multiplication, $\mathbf{0}_{X X}$ as zero and $i d_{X}$ as one. Other examples for semirings are $(\mathbb{N},+, \cdot, 0,1)$ or $(\mathcal{P}(X), \cup, \cap, \emptyset, X)$ for an arbitrary fixed set $X$. 
Definition A.2 (Idempotent semiring). If $x+x=x$ holds for all elements $x$ of a semiring the semiring is called idempotent. On such an idempotent semiring a partial order $\sqsubseteq$ is defined by $x \sqsubseteq y \Leftrightarrow x+y=y$.

The order $x \sqsubseteq y$ means that the choices offered by $x$ are contained in the ones offered by $y$. The operations · and + are $\sqsubseteq$-isotone in both arguments; moreover 0 is the least element w.r.t. $\sqsubseteq$.

The fuzzy relations over a set $X$ form an idempotent semiring and the ordering introduced in Definition 2.4 coincides with the semiring-theoretical one.

\section{A.2 Tests in Semirings}

An important special subset of elements in an idempotent semiring are the socalled tests.

Definition A.3 (Test). An element $p$ of an idempotent semiring with natural order $\sqsubseteq$ is called a test if there is an element $\neg p$, called the complement of $p$, with the properties $p+\neg p=1$ and $p \cdot \neg p=0=\neg p \cdot p$.

This definition is basically due to [MB]; a slightly more liberal definition of tests is given in $[\mathrm{KozT}]$. The definition implies that $p \sqsubseteq 1$ holds for all tests $p$ and that the complement of an element is unique.

Tests are the algebraic counterparts of assertions in programs or predicates characterising subsets of nodes in graphs. Addition and multiplication of tests correspond to their disjunction and conjunction, resp., while $\neg$ corresponds to logical negation. The definition implies that 0 and 1 are tests; they correspond to the everywhere false and to the everywhere true predicate, resp.

Consider a general semiring element $x$ and a test $p$. Then, by isotony of multiplication, $p x \sqsubseteq 1 x=x$. So $p x$ is a part of $x$; it omits all transitions of $x$ that do not start in a point satisfying $p$. This models enforcing the precondition $p$. Similarly, $x p$ removes all transitions of $x$ that do not end in a point satisfying $p$; it enforces $p$ as a postcondition.

This implies the following important property of tests:

Lemma A.4. Let $p, q$ be tests and $a, b$ arbitrary elements in a semiring such that $p q=0$. If $z \sqsubseteq p a$ and $z \sqsubseteq q b$ then $z=0$.

This means that elements with disjoint sets of starting points (remember that the product of tests is their conjunction) are disjoint as well.

The tests in the semiring $\operatorname{Rel}(X, X)$ are precisely the test relations from Definition 2.10.

\section{A.3 Kleene Algebras}

Now we add the concept of finite iteration. 
Definition A.5 (Kleene Algebra). A Kleene algebra [KozKA] is a structure $\left(S,+, 0, \cdot, 1,{ }^{*}\right)$ such that the reduct $(S,+, 0, \cdot, 1)$ is a semiring and the finite iteration operator * satisfies the unfold and induction axioms

$$
1+x x^{*} \leq x^{*}, \quad 1+x^{*} x \leq x^{*}
$$

and the star induction axioms

$$
y+x z \leq z \Rightarrow x^{*} y \leq z, \quad y+z x \leq z \Rightarrow y x^{*} \leq z .
$$

The semiring $\operatorname{Rel}(X, X)$ forms a Kleene algebra under the operation * defined in Section 2.2.

\section{References}

[AMO] R. Ahuja, T. Magnanti, J. Orlin: Network Flows. Prentice Hall 1993

[Glü] R. Glück: Network Flows, Semirings and Fuzzy Relations. Institut für Informatik, Universität Augsburg, Tech. Rep. 2008-01,

http://www .opus-bayern.de/uni-augsburg/volltexte/2008/726/

[GlüI] R. Glück: Import Networks, Fuzzy Relations and Semirings. In R. Berghammer, B. Möller, G. Struth (eds.): Relations and Kleene Algebra in Computer Science PhD Programme Proceedings, RelMiCS10/AKA5, Frauenwörth, Germany, April 7 - April 11, 2008. Institut für Informatik, Universität Augsburg, Technical Report 2008-04, pp. 58-62

[GTT] A. Goldberg, E. Tardos, R. Tarjan: Network Flow Algorithms. In B. Korte, L. Lovasz, H. Prömel, A. Schrijver (eds. ): Algorithms and Combinatorics. Volume 9: Paths, Rows, and VLSI-Layout. Springer 1990, 101-164

[HS] P. Höfner, G. Struth: Automated Reasoning in Kleene Algebra. In F. Pfenning (ed.): Automated Deduction - CADE-21. Lecture Notes in Artificial Intelligence, pp. 279-294, 2007.

[Jun] D. Jungnickel: Graphs, Networks and Algorithms, 2nd ed. Springer 2005

[Kaw] Y. Kawahara: On the Cardinality of Relations. In R.A. Schmidt (Ed.): Relations and Kleene Algebra in Computer Science. Lecture Notes in Computer Science 4136. Springer 2006, 251-265

[KozKA] D. Kozen: A completeness theorem for Kleene algebras and the algebra of regular events. Inf. Comput. 110:2, 366-390 (1994)

$[\mathrm{KozT}]$ D. Kozen: Kleene algebra with tests. ACM Transactions on Programming Languages and Systems, 19(3), 427-443 (1997)

[MB] E. Manes, D. Benson: The Inverse Semigroup of a Sum-Ordered Semiring. Semigroup Forum 31, 129-152 (1985) 